\title{
Mixed Cryoglobulinaemia Vasculitis Treated with Obinutuzumab in a Patient Allergic to Rituximab
}

\author{
Margarida Gaudêncio ${ }^{1}$, Catarina Parente ${ }^{2}$, Ana Catarina Lameiras ${ }^{3}$, António Marinho ${ }^{4,5}$ \\ ${ }^{1}$ Department of Internal Medicine, Hospital Distrital da Figueira da Foz, EPE, Figueira da Foz, Portugal; \\ ${ }^{2}$ Department of Internal Medicine, Hospital Barreiro-Montijo, Lisboa, Portugal; \\ ${ }^{3}$ Department of Internal Medicine, Hospital Fernando Fonseca, Lisboa, Portugal; \\ ${ }^{4}$ Department of Clinical Immunology, Centro Hospitalar do Porto, Porto, Portugal; \\ ${ }^{5}$ Faculty of Medicine, Instituto de Ciências Biomédicas Abel Salazar, Porto, Portugal
}

\section{Doi: 10.12890/2021_003019- European Journal of Case Reports in Internal Medicine - ๑ EFIM 2021}

Received: 20/10/2021

Accepted: 30/10/2021

Published: $22 / 11 / 2021$

How to cite this article: Gaudêncio M, Parente C, Lameiras AC, Marinho A. Mixed cryoglobulinaemia vasculitis treated with obinutuzumab in a patient allergic to rituximab. EJCRIM 2021;8: doi:10.12890/2021_003019.

Conflicts of Interests: The authors declare there are no competing interests.

This article is licensed under a Commons Attribution Non-Commercial 4.0 License

\section{ABSTRACT}

Cryoglobulinaemia is defined as the presence of cryoglobulins in the serum, which are immunoglobulins that reversibly precipitate and form a gel when the temperature is $<37^{\circ} \mathrm{C}$. Autoimmune diseases such as Sjogren's syndrome, systemic lupus erythematosus and rheumatoid arthritis could be associated with mixed cryoglobulinaemia vasculitis (MCV). The treatment of MCV generally consists of glucocorticoids, cytotoxic agents such as cyclophosphamide, plasmapheresis or anti-CD20 monoclonal antibodies including rituximab. Here, we present a case of a 60-year-old woman who developed type II MCV in the context of overlap autoimmune disease and who has been treated with a new anti-CD20 agent, obinutuzumab.

\section{LEARNING POINTS}

- Mixed cryoglobulinaemia can occur in the context of autoimmune diseases such as Sjogren's syndrome, systemic lupus erythematosus and rheumatoid arthritis.

- The treatment generally consists of glucocorticoids, cytotoxic agents such as cyclophosphamide, plasmapheresis or anti-CD20 monoclonal antibodies including rituximab.

- New generations of humanized anti-CD20 monoclonal antibodies such as obinutuzumab have been developed to increase complementdependent cytotoxicity and/or antibody-dependent cellular cytotoxicity, while limiting immunogenicity. These new optimized B-cell depletion strategies could be very interesting and useful in autoimmune disease treatment.

\section{KEYWORDS}

Mixed cryoglobulinaemia, overlap syndrome, autoimmune diseases, remission, vasculitis, obinutuzumab

\section{INTRODUCTION}

Cryoglobulinaemia is defined as the presence of cryoglobulins in the serum, which are immunoglobulins that reversibly precipitate and form a gel when the temperature is $<37^{\circ} \mathrm{C}$ and redissolve if the temperature rises to $>37^{\circ} \mathrm{C}[1]$.

According to Brouet's classification, 3 subtypes of cryoglobulinaemia exist, based on immunoglobulin composition ${ }^{[1]}$. Type I comprises single monoclonal immunoglobulins (most commonly IgM), whereas type II and III are classified as mixed cryoglobulinaemia vasculitis (MCV), 
because they include 2 types of immunoglobulins (IgG and $\lg M^{\text {) }}{ }^{[1]}$. Type II mixed cryoglobulinaemia comprises a combination of monoclonal and polyclonal immunoglobulins, whereas type III comprises polyclonal IgM and IgG ${ }^{[1]}$.

The fundamental mechanism contributing to cryoglobulinaemia vasculitis is aberrant autoantibody production by B cells and B-cell proliferation ${ }^{[1]}$.

The presence of type I is always linked to a B-cell lymphoproliferative disorder ${ }^{[1]}$. By contrast, mixed type II or III are associated with systemic autoimmune diseases, lymphoproliferative disorders and chronic infections such as hepatitis $C$ virus ${ }^{[1]}$.

Autoimmune diseases that are associated with mixed cryoglobulinaemia are Sjogren's syndrome, systemic lupus erythematosus and rheumatoid arthritis ${ }^{[1]}$.

Treatment of MCV generally consists of glucocorticoids, cytotoxic agents such as cyclophosphamide, plasmapheresis or an anti-CD20 monoclonal antibody that triggers cell death of B cells - rituximab ${ }^{[1]}$.

The authors describe a case of MCV in a 60-year-old woman diagnosed with overlap autoimmune disease treated successfully with a new anti-CD20 agent, obinutuzumab.

\section{CASE DESCRIPTION}

A60-year-old woman was diagnosed with overlap autoimmune disease (systemic lupus erythematosus, Sjogren's syndrome and scleroderma) in 2014. Since then, she had been followed up and treated with prednisolone $5 \mathrm{mg}$ /day and methotrexate $10 \mathrm{mg} / \mathrm{week}$ until 2016.

Her medical history was significant for pulmonary tuberculosis (1986 and 2006), pulmonary embolism and toxic hepatitis secondary to rifampicin. She had no significant family history.

In 2016, she was admitted to the internal medicine department with asthenia, anorexia, purpura, arthritis, anaemia, weight loss (>10\%), fever, excessive sweating and adenopathic conglomerates. Further study revealed increased lgG, complement consumption and positive cryoglobulins $(200 \mathrm{mcg} / \mathrm{ml})$ - IgA, IgG and IgM, with polyclonal characteristics but including 2 monoclonal IgG/kappa and IgG/lambda fractions with rheumatoid factor activity.

A digestive endoscopic study was performed and excluded gastrointestinal cancer. Excision of 2 lymph nodes was carried out at different surgical times, excluding infectious or lymphoproliferative disease involvement.

Severe autoimmune progression, refractory to treatment with methotrexate and type II cryoglobulinaemia was therefore assumed. The patient had been proposed for treatment with plasmapheresis and the anti-CD20 agent, rituximab, due to maintenance of clinical complaints, namely fever, arthritis and constitutional symptoms.

She started treatment with rituximab infusion on April 2016, without complications. However, 6 months later, during the second rituximab infusion, the patient developed an allergic reaction and stopped the treatment. After the first infusion, the patient showed no cryoglobulinaemia manifestations and was stable with prednisolone $5-10 \mathrm{mg} / \mathrm{day}$.

Four years later, she developed asthenia, anorexia, arthritis, hypergammaglobulinaemia and high levels in inflammatory tests. This case was discussed as a team and it was decided to start a new anti-CD20 therapy - obinutuzumab, as 1 single dose.

On follow-up with the patient 2 years after the beginning of this treatment there is resolution of the clinical symptoms and laboratory tests and she continues with complete B-cell depletion.

\section{DISCUSSION}

Over the past decades, B-cell depletion with anti-CD20 monoclonal antibodies has constituted a major step forward in the treatment of antibody-mediated autoimmune diseases ${ }^{[2]}$.

However, the proportion of patients who achieve long-term remission depends on the disease and clinical context ${ }^{[2]}$.

The exact mechanisms explaining this diversity are still a matter of debate and they depend on the type of lymphoid cells involved in the autoimmune process and on the microenvironment and tissues in which autoreactive $B$ cells are generated ${ }^{[2]}$.

Anti-CD20 therapy has potential dangers such as reactivation of hepatitis B virus and the development of progressive multifocal leucoencephalopathy ${ }^{[2]}$.

New generations of humanized (ocrelizumab, veltuzumab and obinutuzumab) or fully human (ofatumumab) anti-CD20 monoclonal antibodies have been developed to increase complement-dependent cytotoxicity and/or antibody-dependent cellular cytotoxicity, while limiting immunogenicity ${ }^{[3]}$.

Obinutuzumab is a novel type II glycoengineered humanized anti-CD20 monoclonal antibody that has been developed to address the need for a novel therapy with higher activity than rituximab ${ }^{[4]}$. Thus, the post-translational process used was developed to increase antibodydependent cellular cytotoxicity by enhancing binding affinity for the Fc gamma receptor III expressed on immune effector cells ${ }^{[4]}$. Obinutuzumab has been shown to be superior to rituximab and ofatumumab in inducing direct cell death in malignant $\mathrm{B}$ cells ${ }^{[4]}$. 


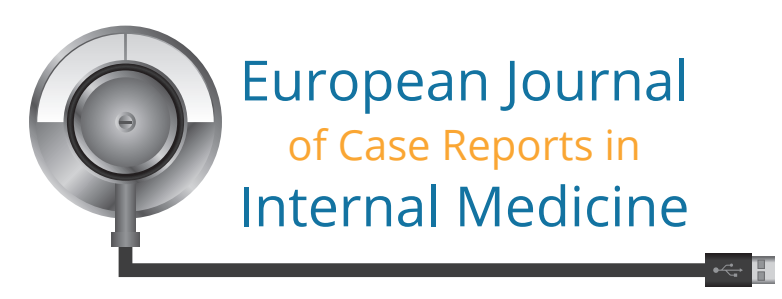

This second generation anti-CD20 agent has been approved by the FDA for chronic lymphocytic leukaemia, follicular lymphoma refractory to rituximab and stage II bulky, III or IV follicular lymphoma ${ }^{[5]}$. This antibody has met its primary endpoint in lupus nephritis in combination with standard of care (NCT02550652) ${ }^{[2]}$.

The authors highlight this case of type II MCV that has been successfully treated with obinutuzumab, after rituximab allergic reaction. The off-label choice of this antibody was based on the mechanism of action. This case may constitute additional evidence of the potential benefits of obinutuzumab in autoimmune diseases such as SLE or MCV; however, further investigation is needed.

\section{REFERENCES}

1. Roccatello D, Saadoun D, Ramos-Casals M, Tzioufas AG, Fervenza FC, Cacoub P, et al. Cryoglobulinaemia. Nat Rev Dis Primers 2018;4(1):11.

2. Crickx E, Weill JC, Reynaud CA, Mahevas M. Anti-CD20-mediated B-cell depletion in autoimmune diseases: successes, failures and future perspectives. Kidney Int 2020;97:885-893.

3. Robak T, Robak E. New anti-CD20 monoclonal antibodies for the treatment of B-cell lymphoid malignancies. BioDrugs 2011;25:13-25.

4. Tobinai K, Klein C, Oya N, Fingerle-Rowson G. A review of obinutuzumab (GA101), a novel type II anti-CD20 monoclonal antibody, for the treatment of patients with B-cell malignancies. Adv Ther 2017;34:324-356.

5. Barnas J, Looney R, Anolik J. B cell targeted therapies in autoimmune disease. Curr Opin Immunol 2019;61:92-99. 